\title{
Study of MiR-451 in the Treatment of Human Gliomas through Regulating the AMPK Pathway Mediated by Bone Mesenchymal Stem Cells
}

\section{Yingwei Zhen}

Department of Neurosurgery, The First Affliated Hospital of Zhengzhou University, Zhengzhou

\section{Cheng Wei}

Tianjin Neurological Institute, Key Laboratory of Post-Neuroinjury Neuro-repair and Regeneration in Central Nervous System, Tianjin Medical University General Hospital,Tianjin

\section{Xiaoyang Zhang}

Tianjin Neurological Institute, Key Laboratory of Post-Neuroinjury Neuro-repair and Regeneration in Central Nervous System, Tianjin Medical University General Hospital,Tianjin

\section{Peng Gao}

Department of Neurosurgery, The First Affliated Hospital of Zhengzhou University, Zhengzhou

\section{Dazhao Peng}

Tianjin Neurological Institute, Key Laboratory of Post-Neuroinjury Neuro-repair and Regeneration in Central Nervous System, Tianjin Medical University General Hospital,Tianjin

\section{Hongbao Guo}

Department of Nephrology, Tianjin Medical University General Hospital, Tianjin

\section{Lei Han}

Tianjin Neurological Institute, Key Laboratory of Post-Neuroinjury Neuro-repair and Regeneration in Central Nervous System, Tianjin Medical University General Hospital,Tianjin

\section{Yang Nan}

Tianjin Neurological Institute, Key Laboratory of Post-Neuroinjury Neuro-repair and Regeneration in Central Nervous System, Tianjin Medical University General Hospital,Tianjin

\section{Dongming Yan}

Department of Neurosurgery, The First Affliated Hospital of Zhengzhou University, Zhengzhou

\section{Yue Zhong ( $\nabla$ zhongyue2457@sina.com )}

Tianjin Neurological Institute, Key Laboratory of Post-Neuroinjury Neuro-repair and Regeneration in Central Nervous System, Tianjin Medical University General Hospital,Tianjin

\section{Research Article}

Keywords: glioma, gene therapy, miR-451, hBMSCs, AMPK 
Posted Date: June 18th, 2021

DOl: https://doi.org/10.21203/rs.3.rs-604846/v1

License: (c) (i) This work is licensed under a Creative Commons Attribution 4.0 International License. Read Full License 


\section{Abstract}

Accumulating evidence has suggested that gene therapy plays critical roles in glioma treatment. Our previous studies demonstrated that miR-451 could be used as a tumor suppressor via targeting CAB39. But the ability for the targeted delivery of miR-451 to glioma was a significant issue. Bone marrow mesenchymal stem cells (BMSCs) have been used for systemic delivery of therapeutic genes to solid tumors. In the present study, hBMSCs were used as gene therapy non-viral vectors of miR-451 to treat glioma cells in vitro and in vivo. First, following the treatment of glioma cells with the culture medium of miR-451-loaded hBMSCs via lentivirus, overexpression of miR-451 in glioma cells suppressed its proliferation, migration and invasion, but enhanced cell apoptosis by targeting CAB39 and the LKB1/AMPK/HIF-1a/VEGF pathways. Secondly, Transwell migration assays further demonstrated the chemotaxis of BMSCs to glioma conditioned media in vitro. Lastly, it was verified that the miR-451-loaded hBMSCs could effectively deliver miR-451 to the tumor region and prolong the survival in xenograft model assays. These results indicated that gene therapy using hBMSCs as vehicles was highly effective in a mice glioma model and therefore hold considerable prospects of clinical transformation.

\section{Introduction}

Glioblastoma (GBM) is the most frequent and aggressive primary brain tumor. Even with the current multimodal therapy, the median survival time of GBM patients was still $<15$ months, and the 5 -year survival rate was $<6 \%(1-3)$. Therefore, it is imperative to explore the pathogenesis of glioma and develop more promising treatment plans.

MicroRNA (miRNA) is a type of non-coding RNA with a length of 19-25 nt that often synergizes with major intracellular signaling pathways to regulate tumor development $(4,5)$. Recent studies have indicated that miR-451 could be abnormally expressed in a variety of malignant tumors, such as glioma(6), lung cancer(7), pancreatic cancer(8), ovarian cancer(9) and digestive system malignant tumors(10). In our previous study, it was found that the expression of miR-451 was decreased in glioma, as compared with adjacent normal tissue, and negatively correlated with pathological grade. It was also shown that miR-451 could inhibit glioma cell proliferation, reduce invasion, and promote cell apoptosis by targeting CAB39 in vitro and in vivo $(11,12)$. These findings suggested that miR-451 is a potential therapeutic target for gliomas.

In recent years, gene therapy is regarded as a new method to eradicate gliomas(13). The specific delivery of gene drugs to tumor sites through vectors could kill tumor cells $(14,15)$. At present, the delivery systems used in gene therapy could be divided into two categories: viral and non-viral vectors(16-18). Bone marrow mesenchymal stem cells (BMSCS) have low immunogenicity, immunomodulatory activity and tropism towards damaged and inflammatory sites, and have the potential to treat a variety of diseases, including cancer $(19,20)$. These advantages, especially the tendency towards tumor regions, encourage the study of BMSCs as vectors of anti-cancer gene drugs. 
In the present study, human BMSCS (hBMSCs) were isolated from human bone marrow. It was found that the supernatant of Lv-miR-451-hBMSC (hBMSCs infected by lenti-virus to overexpress miR-451) could inhibit the proliferation, migration and invasion of glioma cells, and induce cell apoptosis. Mechanically, it was shown that miR-451 transducted via Lv-miR-451-hBMSCs could affect the phenotype of glioma cells by targeting CAB39 and the LKB1/AMPK/HIF-1a/VEGF pathway.

It was also verified that Lv-miR-451-hBMSCs could effectively inhibit the growth of intracranial tumors and prolong the survival in an orthotopic glioma model, which might provide a new therapeutical option for glioma.

\section{Materials And Methods}

\section{Acquisition, isolation and culture of hBMSCs.}

HBMSCs were collected from a patient (male, 32 years old) who underwent femoral shaft fracture surgery in the Tianjin Medical University General Hospital. The experiment was examined and approved by the Ethics Committee of Tianjin Medical University General Hospital, and the relevant ethical agreement was signed. The consent of the patient and their families was obtained in advance when the bone marrow tissue was collected, and the purpose of the experiment was informed.

HBMSCs were isolated by density gradient centrifugation (Ficoll-PaqueTM Plus, GE Healthcare), according to the manufacturer's instructions, and hBMSCs were cultured in low glucose Dulbecco's Modified Eagle's Medium (DMEM-LG; Thermo Fisher Scientific, Inc.), supplemented with 10\% MSCqualified fetal bovine serum (Thermo Fisher Scientific, Inc.), and 1\% penicillin/streptomycin (Thermo Fisher Scientific, Inc.). Human glioma LN229, U87-MG and U251-MG cell lines were long-term subcultured cell lines in our laboratory. The LN229, U87-MG and U251-MG cell lines were subjected to a short tandem repeat (STR) test. The STR result demonstrated that the U87-MG cell line used in the present study matched the U87-MG cell line from ATCC. However, the U87-MG cell line from ATCC may not be the original GBM cell line from the University of Uppsala established in 1968. It has been reported that the U87-MG cell line from ATCC is of CNS origin and is likely to be derived from another patient with glioma, although its source is unknown. Therefore, the aforementioned misidentifications of U87-MG ATCC did not affect the outcomes of the present study. LN229, U87-MG and U251-MG cell lines were cultured in Dulbecco's modified Eagle's medium (DMEM; Thermo Fisher Scientific, Inc.) supplemented with $10 \%$ fetal bovine serum (Thermo Fisher Scientific, Inc.) and were cultured in a humidified $5 \% \mathrm{CO}_{2}$ incubator maintained at $37^{\circ} \mathrm{C}$ (Thermo Scientific 3131).

\section{Oil Red O, Alizarin Red and Von Kossa staining.}

Oil Red O, Alizarin Red and Von Kossa staining were used for lipogenic and osteoblastic staining, respectively. The well-grown third-generation hBMSCs were digested at a regulated concentration of 
$5 \times 10^{4} / \mathrm{ml}$ and inoculated on a 6 -well culture plate with cover glass. The cells were completely adherent to the glass after $24 \mathrm{~h}$.

Lipogenic inducers $(3 \mu \mathrm{g} / \mathrm{ml}$ insulin, $0.45 \mathrm{mM}$ isobutyl methyl xanthine, $1 \mu \mathrm{M}$ Rosiglitazone, $100 \mathrm{nM}$ dexamethasone) (Sigma, US) were used for induction for 3 days(21). The solution was changed twice a week for another 15 days. The cells were then fixed with $4 \%$ paraformaldehyde and observed by oil red 0 staining.

The reagents used for osteogenic induction were $10 \mathrm{mM} \beta$-glycerophosphate (Sigma, US), $50 \mu \mathrm{g} / \mathrm{ml}$ vitamin $\mathrm{C}$ and $10 \mathrm{nM}$ dexamethasone (Sigma, US) (21). After 2-3 days, the culture medium was changed into osteogenic induction medium, and the culture was continued for 3-4 weeks. The changes in cell morphology were visualized on an Olympus upright BX53 microscope. Alizarin Red and Von Kossa staining were used 4 weeks after osteoblast-induced differentiation to observe the calcium deposition of osteoblast differentiation.

\section{Lentiviral infection.}

Lentiviral vectors expressing miR-451 and enhanced green fluorescent protein (EGFP) were constructed from Shanghai GenePharma Co., Ltd (China). The coding sequences were miRNA-451, 5'AAACCGUUACCAUUACUGAGUU-3' and miRNA-NC, 5'-UUCUCCGAAC

GUGUCACGUTT-3'. The well-grown hBMSCs were routinely passed on and incubated until they reached $80 \%$ fusion. One day prior to infection, $2 \times 10^{5} \mathrm{hBMSC}$ were placed on 24-well plates, followed by infection with lentivirus expressing miR-451 $(\mathrm{MOI}=20)$. The cells were incubated for $24 \mathrm{~h}$ and replaced with fresh medium. After $72 \mathrm{~h}$, the fresh medium was changed and the supernatant was collected, filtered, and placed in the refrigerator at $-80^{\circ} \mathrm{C}$ for the subsequent assays.

\section{RNA extraction from cells, extracellular and tissues and qRT- PCR.}

The total RNA was extracted with Trizol reagent from cells, culture supernatants and tissues (Thermo Fisher Scientific, Inc.). Tumor tissue was ground into powder under liquid nitrogen and then transferred to $1.5 \mathrm{ml}$ centrifuge tube and added $1 \mathrm{ml}$ Trizol to centrifuge tube. The subsequent operation was consistent with RNA extraction of cells. As for extracellular RNA extraction, $25 \mathrm{fmol}$ of C. elegans cel-mir-39 standard RNA (Ribobio, Guangzhou, China) was added to each sample as a spike-in control [25 27]. Before isopropanol precipitation, Dr.GenTLE Precipitation Carrier (TAKARA\#9094, RR820A, Takara, Japan) was added as a co-precipitant to promote the yield of extracellular RNA. Then, the RNA $(2 \mu \mathrm{g})$ was reversetranscribed with GoScript reverse transcription system (Promega Corporation) to synthesize cDNA. The GoTaq ${ }^{\circledR}$ qPCR Master Mix (Promega Corporation) was used to detect mRNA expression status on ABI QuantStudio 3, and GAPDH expression was used as an internal control. The miR-451 qPCR Quantitation 
Kit and U6 snRNA qPCR Kit were synthesized by Shanghai GenePharma Co., Ltd. The PCR process followed the steps identified by the Hairpin-it ${ }^{\text {TM }}$ miRNAs quantitative PCR kit. The U6 snRNA quantitative PCR calibration kit was used to detect the PCR primers of U6 snRNA in each group to correct the errors. The oligonucleotide primers used for quantitative PCR were as follows: miRNA-451, 5'GCGGCGCAAAGAATTCTCCT-3' forward and 5'-GTGCAGGGTCCGAGGT-3' reverse; U6, 5'ATTGGAACGATACA GAGAAGATT-3' forward and 5'-GGAACGCTTCACGAATTTG-3' reverse (Shanghai GenePharma Co., Ltd.). The following PCR program was used: $95^{\circ} \mathrm{C}$ for $3 \mathrm{~min}, 40$ cycles of amplification (12 sec at $95^{\circ} \mathrm{C}, 40-60 \mathrm{sec}$ at $62^{\circ} \mathrm{C}$ and $1 \mathrm{~min}$ at $72^{\circ} \mathrm{C}$ ) and a final extension step ( $72^{\circ} \mathrm{C}$ for $2 \mathrm{~min}$ ). Other primers: CAB39, 5'-GAGCATGGCTGTTCTGGAAAAGC-3' forward and 5'- GCTACTGCTTCTG TCTGAGGCT-3' reverse; LKB1, 5'-GGCACCCT CAAAATCTCCGA-3' forward and 5'-CTTGAAGCCGGAGAAGGTGT-3' reverse; AMPK, 5'-CCTGTGA CAAGCACTTACTCC-3' forward and 5'-CTCTGTG GAGTAGCAGTCCC-3' reverse; HIF1a, 5'-CATCAGCTATTTGCGTGTGAGGA-3' forward and 5'-AGCAA TTCATCTGTGCTTTCATGTC-3' reverse; VEGF, 5'-CCTGGTGGACATCTTCCAGGAGTACC - 3' forward and 5'-GAAGCTCATC TCTCCTATGTGCTGGC3 ' reverse; and GAPDH, 5'-TGTGGGCATCAATG GATTTGG-3' forward and 5'-

ACACCATGTATTCCGGGTCAAT-3' reverse. All PCR experiments were performed in triplicate.

\section{MTT assays.}

The glioma cells treated with supernatant as described above were routinely digested and inoculated in a 96-well plate with $2 \times 10^{3}$ cells $(200 \mu \mathrm{l}) /$ well. $20 \mu \mathrm{l} \mathrm{MTT}$ solution ( $5 \mathrm{mg} / \mathrm{ml}$; KeyGEN) was added to each well at $1,2,3,4,5$ and 6 days after $48 \mathrm{~h}$ of culture. The supernatant was discarded and $200 \mu \mathrm{l}$ DMSO was added to each well. Following incubation for $20 \mathrm{~min}$ at room temperature, a microplate reader (BioTek, China) was used to detect the light absorption value of each well at a wavelength of $570 \mathrm{~nm}$. The tumor cell proliferation rate was then calculated.

\section{Plate clonal formation experiment.}

Trypsin was used to digest the cells at the logarithmic growth stage, single-cell suspension was prepared and counted, and the cell density was adjusted to $1 \times 10^{3} / \mathrm{ml}$. Six-well plates were taken, $1 \mathrm{ml}$ cell suspension was added to each well, and the cells were incubated for $12 \mathrm{~h}$ in a $5 \% \mathrm{CO}_{2}$ and $37^{\circ} \mathrm{C}$ saturated humidity environment. Next, $2 \mathrm{ml}$ medium was added to each well, and the liquid was changed once every 3 days, and terminated at $\sim 14$ days. When obvious clones were formed in the petri dish, the culture medium was removed, washed with phosphate-buffered saline (PBS), fixed with $4 \%$ formaldehyde (Merck $\mathrm{KGaA}$ ) for $15 \mathrm{~min}$, discarded with formaldehyde, and dried in air. Next, $2.5 \%$ crystal violet (Merck KGaA) was added for staining for $30 \mathrm{~min}$ and eluted with PBS. The number of clones was counted under the microscope (Olympus, BX53) and the experiment was repeated 3 times.

\section{Flow cytometry.}


Identification of hBMSC surface markers, cell cycle analysis and cell apoptosis analysis were performed by flow cytometry. When the cells were cultured to the third generation in vitro, the cells were washed twice with PBS and digested by $0.25 \%$ trypsin when they reached $~ 80 \%$ confluence. Cell suspensions were collected and centrifuged at $157 \times \mathrm{g}$ for $5 \mathrm{~min}$. After the supernatant was discarded, cell precipitation was resuspended with PBS. The cell concentration was adjusted to $\sim 1 \times 10^{6} / \mathrm{ml}$, and $100 \mu \mathrm{l}$ cell suspension was taken respectively. FITC labeled-CD29 (SAB4700397; Merck KGaA), CD34 (SAB4700703; Merck KGaA), CD44 (SAB4700182; Merck KGaA), CD45 (SAB4700480; Merck KGaA), CD71 (SAB4700518; Merck KGaA) and HLA-DR (SAB4700658; Merck KGaA) antibodies were diluted to the reaction concentration, and staining was performed at room temperature for $30 \mathrm{~min}$ in the dark. Following washing once with PBS, cells were resuspended and detected by flow cytometry.

\section{Cell cycle assay and apoptosis assay.}

After the tumor cells were incubated with the supernatant for $48 \mathrm{~h}$, cells were digested to prepare the cell suspension, which was fully suspended by PBS $(0.01 \mathrm{M}, \mathrm{pH} 7.2)$. The cell suspension was fixed with $75 \%$ ethanol overnight at $4^{\circ} \mathrm{C}$. Next, propidium iodide staining solution was used for staining and detection. Anexin V-FITC Apoptosis Detecion kit (APOAF; Merck KGaA) was used for apoptosis analysis and flow cytometry was performed in accordance with the manufacturer's instructions.

\section{Transwell and wound-healing assays.}

Matrigel was solidified by adding $60 \mu$ diluted Matrigel on the polycarbonate film (diameter, $6.5 \mathrm{~mm}$ ) in a Transwell chamber (BD Biosciences) and left to stand at $37^{\circ} \mathrm{C}$ for $30 \mathrm{~min}$. Single-cell suspension was prepared by serum-free medium, and each group was made into three wells. A total of $1 \times 10^{5}$ cells/well were accurately added in the upper chamber ( $48 \mathrm{~h}$ after culture with the BMSCs supernatant). The cell suspension liquid was accumulated to $100-200 \mu \mathrm{l}$ and placed in an incubator containing $5 \% \mathrm{CO}_{2}$ for 24 $\mathrm{h}$ at $37^{\circ} \mathrm{C}$. After $24 \mathrm{~h}$ of culture, the upper chamber was removed and the surface of the cells was wiped with a wet cotton swab. Cells were then dyed with hematoxylin, thoroughly rinsed with Clearmont seal slice and dried room temperature. Finally, they were observed under an inverted microscope (Olympus, BX53). Three visual fields were randomly selected in and around the center of each membrane to count the number of cells passing through micropores. Each cell line was divided three groups: control, hBMSCs and Lv-miR-451-hBMSCs groups with 3 wells in each group. The glioma cells used in the wound-healing experiment were prepared into a single-cell suspension with an adjusted density of $1 \times 10^{5} / \mathrm{ml}$. Next, 2 $\mathrm{ml} /$ well was inoculated into the 6-well plates and incubated for $24 \mathrm{~h}$. When the cell density reached $90 \%$ of the tiled culture plate, every 3 scratch positions in the 6-well plates were selected and marked on the

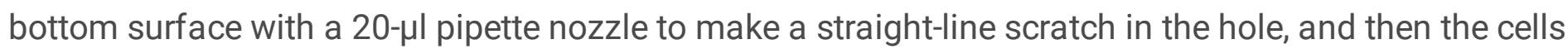
were scraped off with PBS and washed, and then incubated in a constant temperature incubator. Different time points were selected to mark the scratch position of 6-well plates in advance for microscopic observation and measurements, and then the mobility was calculated. 


\section{Chemotactic capacity of BMSCs via the Transwell migration assay in vitro.}

To monitor the dynamic migration of hBMSCs towards chemoattractants, a Boyden Chamber-based assay was performed. Serum-free medium was placed in the top chamber to hydrate the membrane. LvmiR-451-hBMSC and hBMSCs ( $2 \times 10^{4}$ cells) were added in the upper chamber (Corning Costar, USA) and the cells were allowed to settle to the bottom. Conditioned media harvested from U87-MG, was added to the lower chambers. After $24 \mathrm{~h}$ of incubation, the non-migrated cells were scraped from the upper surface of the membrane with cotton swabs. Then, the membrane was stained with crystal violet (Solarbio, Beijing, China). Each sample was tested in triplicate, and a representative field in each well was counted to determine the number of migrated cells. To collect conditioned media, normal culture media was removed from U87 cells and replaced with Alpha-MEM (without FBS) for 48 hours after which the conditioned media was collected for use.

\section{Protein extraction and western blotting.}

The cultured glioma cell lines were further cultured with hBMSC supernatant in incubator at $37^{\circ} \mathrm{C}$. After 48 h, the cultured cells were rinsed with PBS, drained, cultured with $200 \mu$ RIPA cell lysis buffer (Thermo Fisher Scientific, Inc.), transferred to an Eppendorf tube and chilled in a bath for $30 \mathrm{~min}$, and the protein concentration was measured using the bicinchoninic acid (Merck KGaA) method. Dithiothreitol (40 $\mu$ l) and loading buffer $(160 \mu \mathrm{l})$ were added into each tube, boiled for 15-20 min, cooled and centrifuged at $22000 \times \mathrm{g}$ for $15 \mathrm{~min}$, and then the protein supernatant was separated for use.

The same amount of protein was isolated from glioma cells in each group, separated by $10 \%$ SDSpolyacrylamide gel electrophoresis and transferred to polyvinylidene fluoride (PVDF) membranes (EMD Millipore) using a Mini Trans-Blot apparatus (Bio-Rad Laboratories, Inc.). Finally, the PVDF membrane was incubated with PVDF membrane sealing solution (Tiangen Biotech Co.) at $37^{\circ} \mathrm{C}$ for $1 \mathrm{~h}$ and then incubated with mouse antibodies against GAPDH (2118; Cell Signaling Technology, Inc.), CAB39 (2716; Cell Signaling Technology, US), LKB1 (3050; Cell Signaling Technology, Inc.), AMPK (5832; Cell Signaling Technology, Inc.), HIF-1a (14179; Cell Signaling Technology, Inc.), VEGF (Santa Cruz Biotechnology, Inc.). On the next day, the PVDF membrane was washed with a large amount of PBST 3 times for 5 min each, and the membrane was then incubated with HRP-conjugated goat anti-rabbit or anti-mouse secondary antibodies (7074/7076; Cell Signaling Technology, Inc.) at $37^{\circ} \mathrm{C}$ for $1 \mathrm{~h}$ using shaker. The membrane was washed again with PBST 3 times for 5 min each. Finally, the membrane was placed face up in a petri dish and performed the chemiluminescent analysis using the ECL Protein Detection Kit (Pierce). Protein bands were analyzed using the gel imaging system (Syngene Europe).

\section{Animal experiments.}


All mouse experiments were conducted according to protocols approved by the Tianjin Medical University Animal Care and Use Committee and the reporting in the manuscript followed the recommendations in the ARRIVE guidelines. This study was carried out following all the relevant guidelines and regulations (for animals and humans). Specific pathogen-free BALB/C-NU female nude mice aged 4-6 weeks and weighing 15-18 g were purchased from the Institute of Medical Experimental Animals, Chinese Academy of Medical Sciences.

Next, the intracranial glioma model was established. Briefly, U87-MG cells were infected with the luciferase lentivirus (Shanghai GenePharma Co., Ltd.), and the U87-MG cells were suspended in serumfree DMEM medium to make the cell suspension. To generate orthotopic tumors, $5 \times 10^{4}$ U87-MG cells were stereotactically injected into the brains of nude mice in a total volume of $3 \mu \mathrm{l}$ using the previously described stereotactic instrument (Stoelting Co., USA). After 7 days of intracranial tumor cell inoculation, mice were randomly divided into 3 groups: The first group was mainly administered Lv-miR-451-hBMSCs $\left(1 \times 10^{6}\right)(n=9)$ by tail vein, the second group was administered uninfected hBMSCs $(n=9)$ and the third group was just administered PBS $(n=9)$.

In order to obtain tumor growth status in live animals of different treatment groups, the mice were anesthetized and injected intraperitoneally with D-luciferin $(150 \mathrm{mg} / \mathrm{kg}$, beetle luciferin, potassium salt; E1605; Promega Corporation) 15 min prior to imaging with the IVIS imaging system (PerkinElmer, Inc.) for 10-120 sec. Imaging was performed once a week and stopped after 6 weeks. The diet, mental state and physical activity of the nude mice were observed every day, and the weight change of the three groups was monitored once every 3 days. The weight change curve was plotted at the end of the experiment. Six weeks after implantation, three animals from each group were sacrificed, and the brains were collected for H\&E, TUNEL staining and immunohistochemistry (IHC). The remaining six mice in each group were used for survival analysis.

\section{Immunofluorescence analysis}

Lv-miR-451-hBMSC $\left(1 \times 10^{6}\right)$ were injected into the tail veins of the intracranial glioma -bearing mice $(\mathrm{n}=$ $3)$. The mice were sacrificed after 24 hours post-injection, and the tumors were removed and snap-frozen for immunofluorescence staining. The slides from frozen tumor tissues were incubated with DAPI. The slides were rinsed several times to remove all free DAPI, and then viewed under a fluorescent confocal microscope (FV1200, Olympus).

\section{H\&E staining and IHC.}

In order to conduct histological analysis, tumor tissues were fixed in $10 \%$ neutral buffered formalin for $\mathrm{H} \& \mathrm{E}$ staining and IHC. The paraffin-embedded brain sections were treated with xylene and then hydrated with ethanol and distilled water. Next, they were washed with PBS for 3 times, 5 min each time, then dyed with hematoxylin for $5 \mathrm{~min}$, and washed with distilled water. The slices were stained with eosin for 2 min, 
and rinsed with distilled water. Following normal dehydration and neutral glue sealing, the slices were observed and photographed under an Olympus upright BX53 microscope.

For IHC, paraffin sections were dewaxed in xylene I and II each $15 \mathrm{~min}$, anhydrous ethanol I and II, 95\% ethanol, and $80 \%$ ethanol and distilled water every $15 \mathrm{~min}$. Next, antigen retrieval was performed using sodium citrate $(0.01 \mathrm{M}, \mathrm{pH}=6)$ buffer at $97^{\circ} \mathrm{C}$ for $20 \mathrm{~min}$. Slides were then washed in PBS prior to incubation in $3 \% \mathrm{H}_{2} \mathrm{O}_{2}$ for 5 min to block endogenous peroxidases. After washing in PBS, slides were blocked with blocking serum for $30 \mathrm{~min}$ at room temperature. Next, the slides were incubated at $4^{\circ} \mathrm{C}$ overnight in a 1:100 dilution with primary antibodies against CAB39 (2716; Cell Signaling Technology), LKB1 (3050; Cell Signaling Technology), AMPK (5832; Cell Signaling Technology), HIF-1 a (14179; Cell Signaling Technology), VEGF (MA5-13182; Thermo Fisher Scientific) before incubated with a biotinlabeled secondary antibody (1:100 dilution) for $1 \mathrm{~h}$ at $37^{\circ} \mathrm{C}$, followed by incubation with $\mathrm{ABC}$-peroxidase and diaminobenzidine (DAB). The slides were then counterstained with hematoxylin and mounted.

\section{Tunnel assay.}

The TUNEL apoptosis kit (Roche Diagnostics) was used to detect the apoptosis of tumor cells in each group in situ. The paraffin sections were routinely hydrated, and the endogenous peroxidase was inactivated by $\mathrm{H}_{2} \mathrm{O}_{2}$ at room temperature for $30 \mathrm{~min}$. Then, the paraffin sections were washed with PBS (0.01 M, pH 7.2-7.4) 3 times for 5 min. The cover glass was soaked in permeable solution at room temperature for 30 min $(0.1 \%$ Triton X-100, $0.1 \%$ sodium citrate, freshly prepared). The labeled solution was added with $20-25 \mu \mathrm{l}$ in a wet box at $4^{\circ} \mathrm{C}$ overnight, and then washed with PBS $(0.01 \mathrm{M}, \mathrm{pH} 7.2-7.4)$ 3 times for $5 \mathrm{~min}$. DAPI was diluted with $0.01 \mathrm{M} \mathrm{PBS}(\mathrm{pH} 7.2-7.4)$ at 1:500 and the nuclei were restrained for $5 \mathrm{~min}$. And the paraffin sections were washed with PBS $(0.01 \mathrm{M}, \mathrm{pH} 7.2 \sim 7.4) 3$ times for $5 \mathrm{~min}$, and covered with $0.5 \mathrm{M} \mathrm{Na}_{2} \mathrm{CO}_{3}-50 \%$ glycerol. IX81 fluorescence microscope was used for observation and photography.

\section{Statistical analysis.}

SPSS 19.0 statistical software (IBM Corp.), $\chi^{2}$ test and one-way ANOVA were used for statistical analysis, and $P<0.05$ was considered to indicate a statistically significant difference. One-way ANOVA was used to analyze the difference in the photon number in the in vivo images of the intracranial glioma model, as well as the weight change of mice. Kaplan-Meier survival analysis was used for survival, and the log-rank test was performed.

\section{Results}

\section{Morphological observation of hBMSCs.}

Following density gradient centrifugation, hBMSCs were extracted and grew adherent, presenting a spindle or polygon shape (Fig. S1A). Morphological observation of the 4th-generation cultured hBMSCs 
showed that hBMSCs had the relatively uniform morphology and vigorous growth, and were closely arranged in a long spindle and whirlpool shape (Fig. S1B).

\section{Related characteristics of hBMSCs.}

The proliferation capacities of hBMSCs were confirmed for a total of 9 days at the 1st (P1), 4th (P4) and 8th (P8) generations. The growth curve of the hBMSCs in P4 was similar to an "S". Cell proliferation was relatively slow from days $1-3$, which was the incubation period. Rapid proliferation and active cell division occurred from days $3-5$, and entered the logarithmic growth period. These results also revealed the greater proliferation capacity of hBMSCs in the P1, P4 and P8 generations (Fig. S1C and S1D).

Next, flow cytometry was performed to analyze the expression of CD29, CD34, CD44, CD45, CD71 and HLA-DR surface markers in 3rd-generation BMSCs. The positive rate of CD29, CD44 and CD71 was 90.9\%, 98.9\% and $99.0 \%$ respectively, and the counter part for the negative marker HLA-DR, CD34 and CD 45 was $0.412 \%, 0.136 \%, 2.41 \%$ respectively. The tests verified that the purity of the extracted hBMSCs was relatively high (Fig. S1E and S1F).

The capability of differentiation into osteoblasts and adipocytes is a crucial biological feature of hBMSCs. Specific culture medium was used to induce lipogenesis for 2 weeks, a large number of lipid droplets appeared in the cells via Oil red $\mathrm{O}$ staining (Fig. S2A). After the culturing with osteogenic medium for 4 weeks, mineralized nodules were formed and could be observed in hBMSC cells via Alizarin red and Von Kossa staining (Fig. S2B). These results indicated that the purified cells were hBMSCs with an active growth, relatively uniform morphology and great differentiation function.

\section{Infection efficiency of hBMSCs with lentiviruses.}

Our previous study found that miR-451 inhibited the mTOR/HIF-1a/VEGF pathway by targeting CAB39, and inhibited the proliferation and invasion of glioma cells in vitro and in vivo(12).

Therefore, lentiviruses were first used to transduct miR-451 to hBMSCs. HBMSCs showed no significant changes in cell morphology following infection with Lv-miR-451, as compared with those without infection (Data not shown). We then used RT-PCR to detect the expression level of miR-451 in hBMSCs following infection. As compared with the control group, it was found that the expression level of miR-451 was significantly upregulated following treatment with Lv-miR-451, which was 92.41 times (Fig. 1A). In addition, the viability of these hBMSCs cells transducte with miR-451 was not changed and their proliferation ability was identical to that of the hBMSCs (Fig. 1B).

\section{HBMSCs mediate miR-451 in the treatment of glioma by regulating the AMPK signaling pathway in vitro.}


After hBMSCs were infected with lentivirus, miR-451 was sustainable, stably and efficiently expressed within the hBMSCs. The expression level of miR-451 was detected in the culture supernatant of hBMSCs and Lv-miR-451-hBMSCs group and it was found that the expression level of miR-451 was significantly upregulated in the culture supernatant of Lv-miR-451-hBMSCs group compared with the hBMSCs (Fig. $1 C)$. Then, the supernatant medium was collected from infected hBMSCs to culture glioma cell lines. The expression of miR-451 was detected in glioma cell lines to verify whether hBMSCs cells could be used as non-viral vectors for miR-451 transduction. It was shown that the expression level of miR-451 was significantly increased in glioma cell lines treated with the supernatant of Lv-miR-451-hBMSCs, as compared with the control group (Fig. 1D).

After the increased expression of miR-451, the MTT and colony-formation assay were conducted on glioma cells to analyze the changes in cell proliferation activity. Following culture with the supernatant of hBMSCs, the growth of glioma cells did not show significant inhibition. However, a significant inhibitory effect was observed in the growth of glioma cells treated with the supernatant of Lv-miR-451-hBMSCs (Fig. 1E). We then examined the colony-formation ability of glioma cells with miR-451 overexpression. Flow cytometry was used to analyze the effect of miR-451 upregulation on the cell cycle of glioma cells. The results showed that the glioma cells treated with the supernatant of Lv-miR-451-hBMSCs were significantly increased at the G0/G1 phase, significantly decreased at the G2/M phase, and arrested at the G0/G1 phase, as compared with the control group (Fig. 2A-C). This indicated that the proliferation rate of glioma cells with miR-451 overexpression was inhibited.

The Transwell assay results also showed that the invasive ability of the glioma cells treated with the supernatant of Lv-miR-451-hBMSCs was significantly inhibited, as compared with that in the control group (Fig. 3A-C). A cell scratch experiment was then performed and the mobility ability of glioma cells treated with the supernatant of Lv-miR-451-hBMSCs was significantly decreased, as compared with that in the control and hBMSCs groups (Fig. 3D).

Subsequently, Annexin V/PI staining was used to detect apoptosis. The apoptosis rate of U87-MG cells in the control and hBMSCs groups was $4.45 \pm 0.11 \%$ and $6.07 \pm 0.09 \%$, respectively, and the apoptotic rate of U87-MG cells in the Lv-miR-451-hBMSCs group was significantly increased to $13.91 \pm 0.21 \%(P<0.01)$. The apoptosis rate of U251-MG cells in the control and hBMSCs groups was $0.75 \pm 0.11 \%$ and $0.59 \pm$ $0.13 \%$, respectively, and the apoptotic rate of U251-MG cells in the Lv-miR-451-hBMSCs group was significantly increased to $4.26 \pm 0.17 \%(P<0.01)$. The apoptotic rate of LN229 cells in the control and hBMSCs groups was $7.25 \pm 0.12 \%$ and $5.59 \pm 0.21 \%$, respectively, and the apoptotic rate of LN229 cells in the Lv-miR-451-hBMSCs group was significantly increased to $15.49 \pm 0.40 \%(P<0.01)$ (Fig. 4A-C).

Previous studies have shown that miR-451 could regulate the LKB1/AMPK/PI3K/Akt and mTOR/HIF$1 \mathrm{a} / \mathrm{VEGF}$ pathways by targeting CAB39 to act as a tumor suppressor. The changes in the expression of CAB39, LKB1, AMPK, HIF-1 and VEGF in the LKB1/AMPK/HIF-1 a/VEGF pathway were then detected following the upregulation of miR-451 in glioma cells via hBMSCs. The results showed that following the overexpression of miR-451 via Lv-miR-451-hBMSCs, the mRNA and protein expression levels of CAB39, 
LKB1, AMPK, HIF-1a and VEGF were significantly decreased, as compared with the control and hBMSCs groups (Fig. 4D and E).

The above experimental results showed that the supernatant of Lv-miR-451-hBMSCs significantly promoted the expression of miR-451 in glioma cells, and inhibited proliferation. It was also found that miR-451 might affect the above phenotypes by regulating the LKB1/AMPK/HIF-1a/VEGF pathway.

\section{Migration of BMSCs to glioma cells in vitro}

The Transwell system was used to monitor the migration of Lv-miR-451-hBMSCs, and hBMSCs towards conditioned media from the glioma cell line (U87-MG). The rates of hBMSC lines migration towards U87MG was found to be comparable for Lv-miR-451-hBMSCs, and there was minimal migration of hBMSCs towards free media (Fig. 4F).

\section{HBMSCs mediate miR-451 in the treatment of glioma by regulating AMPK signaling in vivo.}

In order to further evaluate the anti-tumor effect of Lv-miR-451-hBMSCs in vivo, we constructed the U87 glioma orthotopic xenograft model. On the 7th day of the experiment, the imaging showed intracranial fluorescent signals in all mice, which had a relatively uniform intensity and tumor formation rate of $100 \%$ in vivo. The mice were then randomly divided into three groups, namely the Lv-miR-451-hBMSCs, hBMSCs and control groups. The treatment regimen was single-dose injected for one time through tail vein injection.

After 2 weeks, intracranial intensity among the Lv-miR-451-hBMSCs, control and hBMSCs groups was not significantly different. Subsequently, the difference in signal intensity between the Lv-miR-451-hBMSCs group and the latter two tumors became more and more significant. At the 42nd day of treatment, the signal intensity in the Lv-miR-451-hBMSCs group was $4.42 \times 10^{4} \pm 1.68 \times 10^{3}$, lower than the initial signal intensity, while the intracranial signal intensity in the latter two groups showed a significant increase (Fig. $5 A$ and $B)$.

In order to track migration of Lv-miR-451-hBMSCs, hBMSCs were labeled by EGFP. After 24h delivery, the brain was isolated and collected to observe EGFP signals in the brain sections. Figure 6A clearly shows that there were very stronger and big green fluorescence spot, which was located at the xenograft tumor area in the hBMSC and Lv-miR-451-hBMSCs groups. Then, the expression levels of miR-451 in the control, hBMSCs and Lv-miR-451-hBMSCs groups were detected and showed that Lv-miR-451-hBMSCs could deliver miR-451 to the tumor region (Fig. 6B).

Figure S2C shows the weight change curves of mice treated with different treatments. The mouse body weight was $18.60 \pm 0.38,18.58 \pm 0.37$ and $20.40 \pm 0.42 \mathrm{~g}$, in control, hBMSCs and Lv-miR-451-hBMSCs groups respectively, on the 18th day of treatment. As compared with the first two groups, body weight and 
survival time in the Lv-miR-451-hBMSCs group was significantly increased $(P<0.01$; Figure S2C and Fig. $5 C)$.

Finally, we analyzed apoptosis and protein expression in full-brain sections from control and Lv-miR-451hBMSCs groups (Fig. S2D). The TUNEL test showed that the apoptosis of tumor cells in the Lv-miR-451hBMSCs group was significantly increased, as compared with that in the other two groups. This result further verified that the upregulation of miR-451 promoted the apoptosis of U87-MG tumor cells in vivo (Fig. 6C). In addition, IHC of tumor tissues indicated that, in the LV-miR-451-hBMSCs treatment group, the expression of CAB39, LKB1, AMPK, HIF-1a, VEGF and Ki67 were decreased, while little change was observed in the hBMSC and control groups, which confirmed that miR-451 may inhibit glioma by regulating CAB39/LKB1/AMPK/HIF-1a/VEGF pathway in glioma (Fig. 6D).

The above results indicated that the transduction of miR-451 to U87-MG glioma cell line via hBMSCs as vectors could effectively inhibit the growth of intracranial tumors, prolong survival and improve prognosis. It was also shown that the effectiveness of hBMSCs as carriers and the convenience of intravenous administration provided an effective reference value for subsequent basic and clinical studies.

\section{Discussion}

MiRNAs are a kind of small non-coding RNAs comprised of 22-25 nucleotides, which are highly implicated in regulating pathophysiologic mechanisms via repressing target gene expression(22). However, effectively and specifically delivering miRNA to the lesion site of glioma is still being extensively studied. The tumor-homing property of hBMSCs renders them as attractive cell vehicles for the specific delivery of therapeutic molecules to tumor cells $(23,24)$. However, the application of hBMSCs in the treatment of glioma remains largely unknown. Our previous studies have found that miRNA- 451 has been considered to be highly effective for glioma therapy through the mTOR/HIF-1a/VEGF signaling pathway by targeting CAB39(25). In view of the excellent function of hBMSCs and their obvious homing and tropism to tumor cells, we evaluated the therapeutic effect of hBMSCs loaded with miR-451 in vivo and in vitro glioma models.

In the present study, hBMSCs from human femur are successfully isolated, cultured and identified. The morphology of adherent hBMSCs was then observed, and their surface antigens were identified by flow cytometry to determine whether the cells cultured were hBMSCs (Fig. S1B, E and F). HBMSCs were infected by lentivirus to construct the stable overexpression of miR-451 (Lv-miR-451-hBMSCs), and then its supernatant medium was co-cultured with glioma cell lines. Subsequently, the expression of miR-451 in the glioma cell lines was significantly increased, indicating that miR-451 could be transducted to glioma cell lines via hBMSCs (Fig. 1D).

Moreover, following the delivery of miR-451 to the glioma cell lines via hBMSCs, the proliferation, migration and invasion abilities of glioma cells were suppressed and the apoptosis was induced (Fig. 1E, $2 A-C, 3 A-D$ and $4 A-C$ ), which is consistent with the findings of our previous study that miR-451 
suppresses the proliferation and invasion of glioma cells by targeting CAB39. CAB39 is a component of the trimeric LKB1-STRAD-MO25 complex and its role is to stabilize the binding of STRAD to LKB1. LKB1 is activated through its interaction with STRAD and MO25, then LKB1 complexed activated AMPK by phosphorylating Thr172. AMPK activated Akt by regulating PI3K. Therefore, miR-451 carried by hBMSCs plays a pivotal role in the regulatory process by suppressing CAB39 expression, activating the LKB1/AMPK/PI3K/AKT signaling pathway, and subsequently inhibits cell proliferation and induces apoptosis of glioma cells.

Finally, the tumor homing and therapeutic abilities of hBMSCs were further analyzed in the orthotopic glioblastoma mouse model. Our study in vivo showed that EGFP-labeled hBMSCs injected from the tail vein can migrate and delivery miR-451 to the tumor, indicating the strong ability of hBMSCs to track glioma cells (Fig. 6A and B). A significant decrease in tumor growth and a subsequent increase in survival were observed when mice bearing glioblastoma were treated with intravenous Lv-miR-451-hBMSCs injection (Fig. 5A-C and Fig. S2C). Immunohistochemistry in tumor xenograft model was performed to show that over-expression of miR-451 led to a marked downregulation of CAB39, LKB1, AMPK, HIF-1a, VEGF, and Ki67 (Fig. 6D). This evidence, both in vitro and in vivo, implied that miR-451 can suppress cell proliferation in human glioma through the LKB1/AMPK/PI3K/AKT pathway.

The significant tumor tropism and therapeutic effect of miR-451-loaded hBMSCs to glioma cells has been observed both in vitro and in vivo. However, we still cannot elaborate the mediators of intercellular communication between hBMSCs and glioma cells. Accumulating evidence have reported that hBMSCderived EVs are the major mediators of cell-cell communication because they can be captured by neighboring cells. Thus, the mechanisms of miR-451 in hBMSCs delivery to glioma cells is under study in our lab. Collectively, hBMSCs might be a promising therapeutic gene delivery vehicle for tumor because of a good tropism for malignant glioma.

\section{Declarations}

\section{Acknowledgements}

This study was supported by the grants (Nos. 81773187, and 81572496) from the National Nature Science Foundation of China, and the Tianjin Natural Science Foundation, China (Grant no. 18JCZDJC98600). Support was also received from the Tianjin High School Program for Young and Middle-aged Talents Backbone and the Tianjin Young Medical Talents Program.

\section{Availability of data and materials}

Not applicable.

\section{Authors' contributions}


Y.Zhong, DM.Yan and Y.Nan conceived and designed the study; YW.Zhen and performed the majority of the experiments. C.Wei interpreted datas and co-wrote the paper; XY.Zhang, HB.Guo and Dazhao Peng wrote part of the manuscript; P.Gao also gave academic guidance during the process of experimental design. L.Han gave guidance and constructive suggestions in writing the paper. All authors read and approved the final manuscript.

\section{Ethics approval and consent to participate}

The present study was approved by the Ethics Committee of Tianjin Medical University.

\section{Patient consent for publication}

Not applicable.

\section{Competing interests}

The authors declare that they have no competing interests.

\section{References}

1. Shergalis A, Bankhead AR, Luesakul U, Muangsin N and Neamati N: Current Challenges and Opportunities in Treating Glioblastoma. Pharmacol Rev 70: 412-445, 2018.

2. Eskilsson E, Rosland GV, Solecki G, Wang Q, Harter PN, Graziani G, Verhaak R, Winkler F, Bjerkvig R and Miletic H: EGFR heterogeneity and implications for therapeutic intervention in glioblastoma. Neuro Oncol 20: 743-752, 2018.

3. Bovenberg MS, Degeling MH and Tannous BA: Advances in stem cell therapy against gliomas. Trends Mol Med 19: 281-91, 2013.

4. Lu TX and Rothenberg ME: MicroRNA. J Allergy Clin Immunol 141: 1202-1207, 2018.

5. Lin S and Gregory RI: MicroRNA biogenesis pathways in cancer. Nat Rev Cancer 15: 321-33, 2015.

6. Liu B, Cao W and Ma H: Knockdown of IncRNA LSINCT5 suppresses growth and metastasis of human glioma cells via up-regulating miR-451. Artif Cells Nanomed Biotechnol 47: 2507-2515, 2019.

7. Liu Y, Li H, Li LH, Tang JB and Sheng YL: Mir-451 inhibits proliferation and migration of non-small cell lung cancer cells via targeting LKB1/AMPK. Eur Rev Med Pharmacol Sci 23: 274-280, 2019.

8. Guo R, Gu J, Zhang Z, Wang Y and Gu C: MiR-451 Promotes Cell Proliferation and Metastasis in Pancreatic Cancer through Targeting CAB39. Biomed Res Int 2017: 2381482, 2017.

9. Wu RL, Ali S, Bandyopadhyay S, Alosh B, Hayek K, Daaboul MF, Winer I, Sarkar FH and Ali-Fehmi R: Comparative Analysis of Differentially Expressed miRNAs and their Downstream mRNAs in Ovarian Cancer and its Associated Endometriosis. J Cancer Sci Ther 7: 258-265, 2015. 
10. Shen Y, Gong JM, Zhou LL and Sheng JH: MiR-451 as a new tumor marker for gastric cancer. Oncotarget 8: 56542-56545, 2017.

11. Nan Y, Han L, Zhang A, Wang G, Jia Z, Yang Y, Yue X, Pu P, Zhong Y and Kang C: MiRNA-451 plays a role as tumor suppressor in human glioma cells. Brain Res 1359: 14-21, 2010.

12. Tian $Y$, Nan $Y$, Han L, Zhang A, Wang G, Jia Z, Hao J, Pu P, Zhong Y and Kang C: MicroRNA miR-451 downregulates the PI3K/AKT pathway through CAB39 in human glioma. Int J Oncol 40: 1105-12, 2012.

13. Kane JR, Miska J, Young JS, Kanojia D, Kim JW and Lesniak MS: Sui generis: gene therapy and delivery systems for the treatment of glioblastoma. Neuro Oncol 17 Suppl 2: ii24-ii36, 2015.

14. Foreman PM, Friedman GK, Cassady KA and Markert JM: Oncolytic Virotherapy for the Treatment of Malignant Glioma. Neurotherapeutics 14: 333-344, 2017.

15. Przystal JM, Waramit S, Pranjol M, Yan W, Chu G, Chongchai A, Samarth G, Olaciregui NG, Tabatabai $\mathrm{G}$ and Carcaboso AM, et al.: Efficacy of systemic temozolomide-activated phage-targeted gene therapy in human glioblastoma. Embo Mol Med 11: 2019.

16. Hu YL, Fu YH, Tabata Y and Gao JQ: Mesenchymal stem cells: a promising targeted-delivery vehicle in cancer gene therapy. J Control Release 147: 154-62, 2010.

17. Zhang Q, Xiang W, Yi DY, Xue BZ, Wen WW, Abdelmaksoud A, Xiong NX, Jiang XB, Zhao HY and Fu P: Current status and potential challenges of mesenchymal stem cell-based therapy for malignant gliomas. Stem Cell Res Ther 9: 228, 2018.

18. Murphy AM and Rabkin SD: Current status of gene therapy for brain tumors. Transl Res 161: 339-54, 2013.

19. Chen R, Lee WY, Zhang XH, Zhang JT, Lin S, Xu LL, Huang B, Yang FY, Liu HL and Wang B, et al.: Epigenetic Modification of the CCL5/CCR1/ERK Axis Enhances Glioma Targeting in Dedifferentiation-Reprogrammed BMSCs. Stem Cell Rep 8: 743-757, 2017.

20. Xu G, Guo Y, Seng Z, Cui G and Qu J: Bone marrow-derived mesenchymal stem cells co-expressing interleukin-18 and interferon-beta exhibit potent antitumor effect against intracranial glioma in rats. Oncol Rep 34: 1915-22, 2015.

21. Elsafadi M, Shinwari T, Al-Malki S, Manikandan M, Mahmood A, Aldahmash A, Alfayez M, Kassem M and Alajez NM: Convergence of TGFbeta and BMP signaling in regulating human bone marrow stromal cell differentiation. Sci Rep 9: 4977, 2019.

22. Rupaimoole R, Calin GA, Lopez-Berestein G and Sood AK: miRNA Deregulation in Cancer Cells and the Tumor Microenvironment. Cancer Discov 6: 235-46, 2016.

23. Ma Z, Cui X, Lu L, Chen G, Yang Y, Hu Y, Lu Y, Cao Z, Wang Y and Wang X: Exosomes from glioma cells induce a tumor-like phenotype in mesenchymal stem cells by activating glycolysis. Stem Cell Res Ther 10: 60, 2019.

24. Xu G, Guo Y, Seng Z, Cui G and Qu J: Bone marrow-derived mesenchymal stem cells co-expressing interleukin-18 and interferon-beta exhibit potent antitumor effect against intracranial glioma in rats. Oncol Rep 34: 1915-22, 2015. 

Proliferation and Invasion Through the mTOR/HIF-1alpha/VEGF Signaling Pathway by Targeting CAB39. Hum Gene Ther Clin Dev 29: 156-166, 2018.

\section{Figures}

A

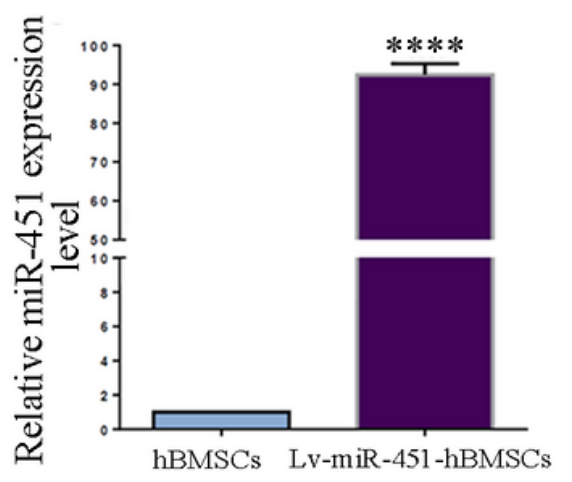

$\mathrm{D}$

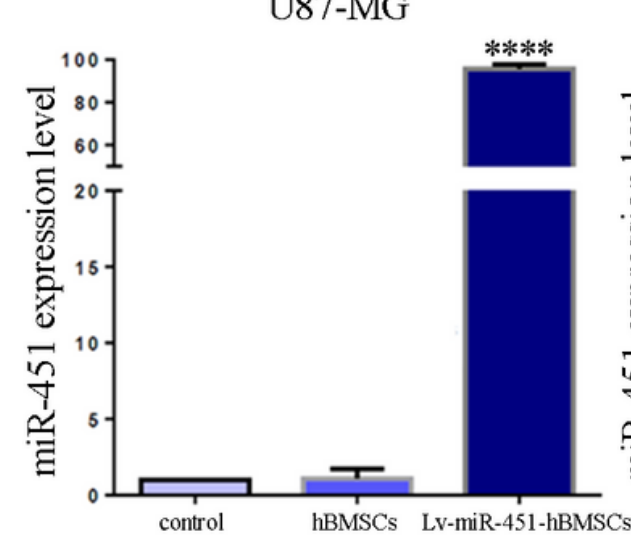

E

U87-MG

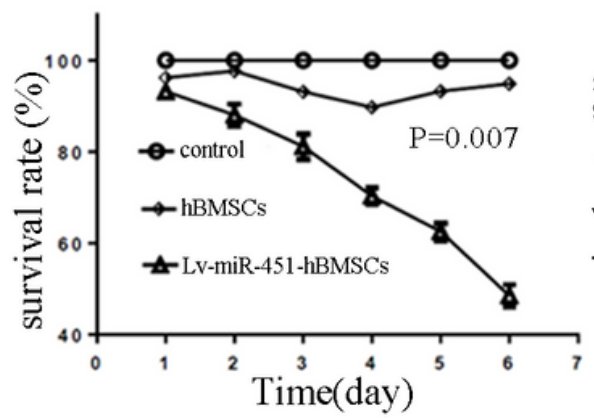

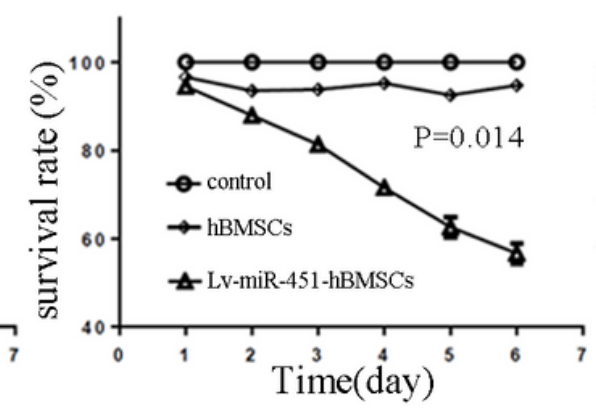

$\mathrm{C}$

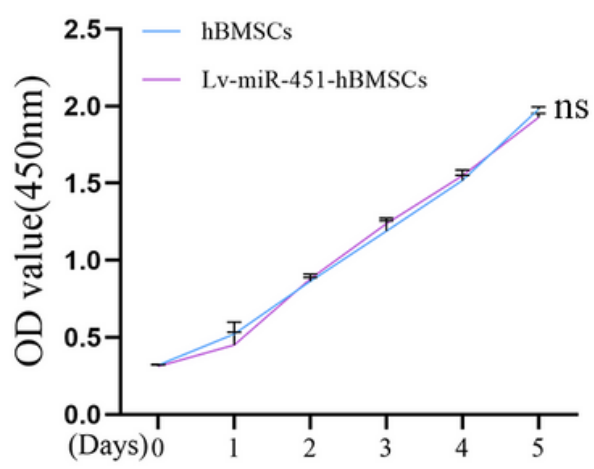

U251-MG

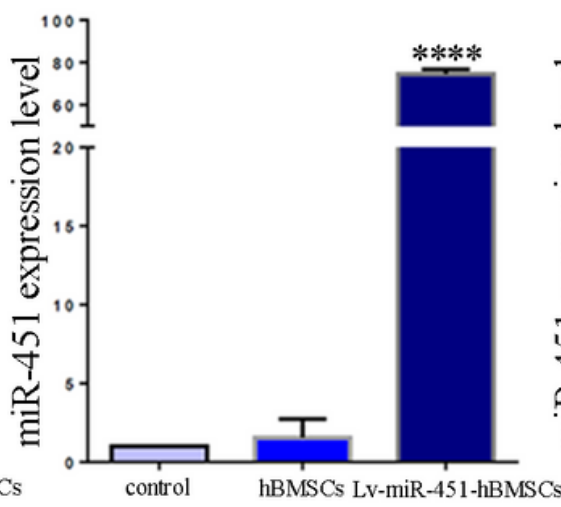

U251-MG

LN229

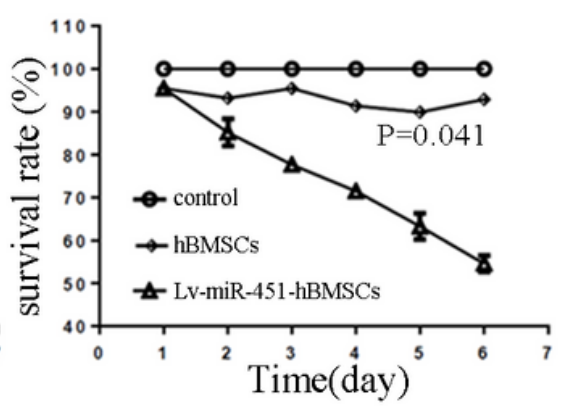


miR-451 was overexpressed in hBMSCs and transported into glioma cells. (A) mRNA level of miR-451 in hBMSCs infected by Lv-miR-451 measured by RT-qPCR. P<0.0001, compared with that of hBMSC cells.

(B) Cell viability of hBMSCs and miR-451-loaded hBMSCs was analyzed via MTT assay. (C) mRNA expression level of miR-451 in culture medium from hBMSCs and miR-451-loaded hBMSCs measured by RT-qPCR, ${ }^{* \star *}$ p $<0.0001$ compared with that of hBMSCs. (D) mRNA expression level of miR-451 after coculture of glioma cells with culture medium of miR-451-loaded hBMSCs measured by RT-qPCR, $\star \star \star * p<0.0001$ compared with the glioma cells co-culture with the medium of hBMSCs. (E) Cell viability from different treatment groups of glioma cells was detected by MTT assay. (The results were measurement data, which were expressed as mean \pm standard deviation. The experiment was repeated for three times independently)
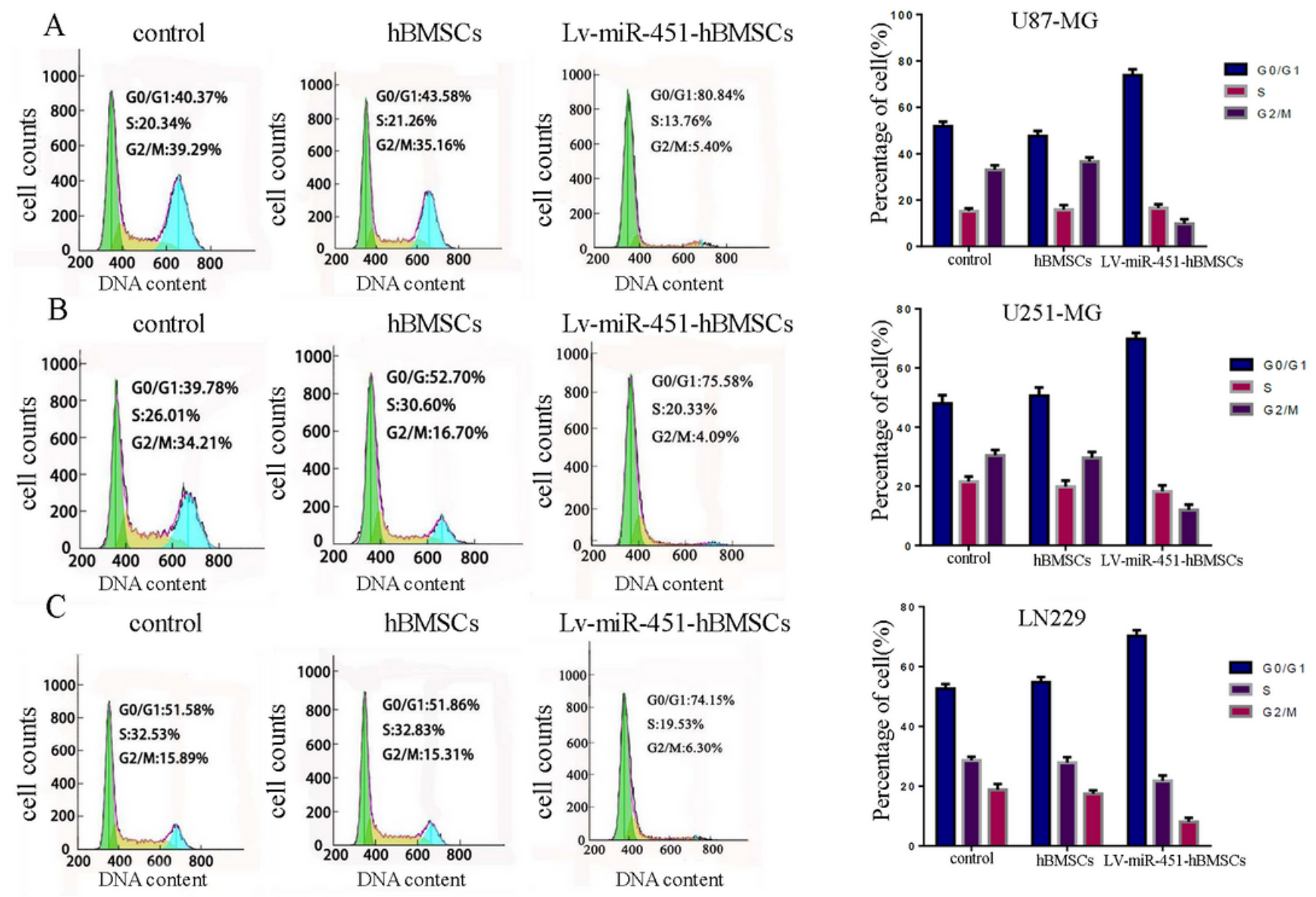

\section{Figure 2}

The culture medium of miR-451-loaded hBMSCs inhibits cell cycle progression of glioma cells. (A-C) Cell cycle analysis in different treatment groups of glioma cells. (The results were measurement data, which were expressed as mean \pm standard deviation. The experiment was repeated for three times independently. $\left.{ }^{*} \mathrm{P}<0.01\right)$ 
A

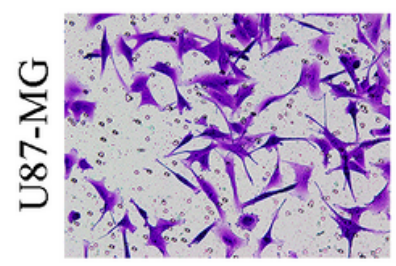

B
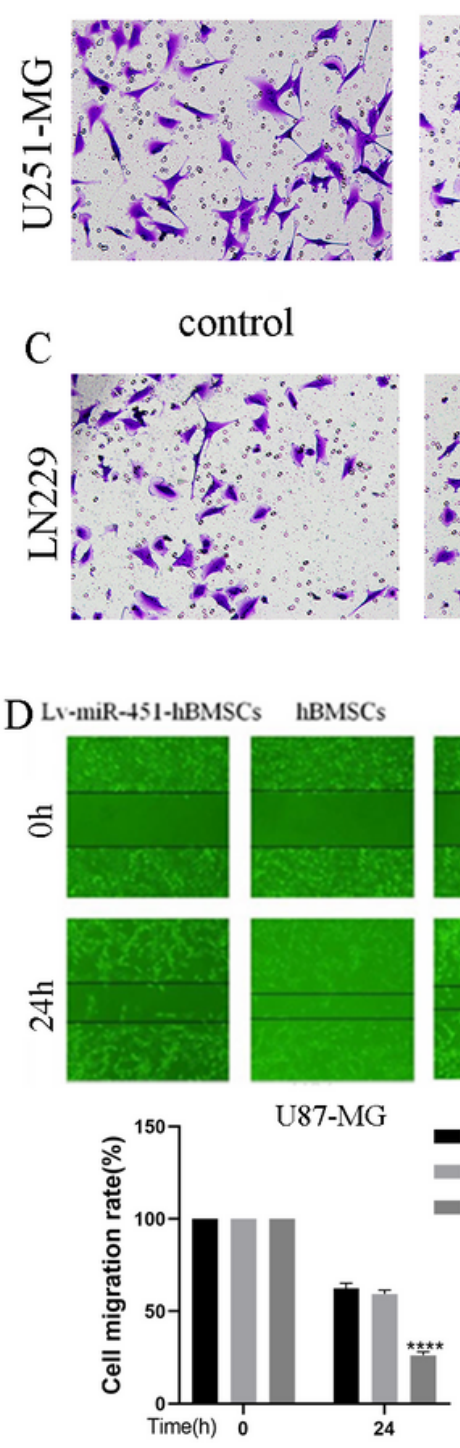

hBMSCs

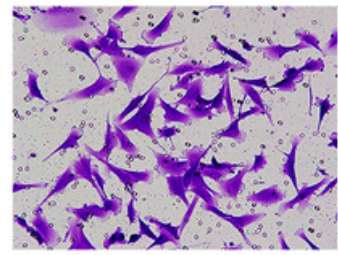

hBMSCs

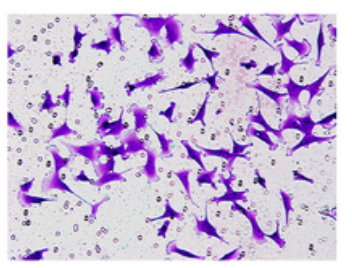

hBMSCs

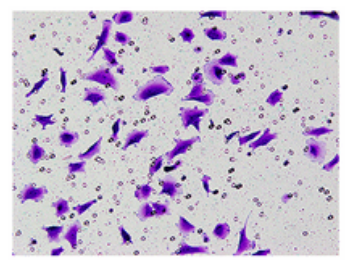

Lv-miR-451-hBMSCs

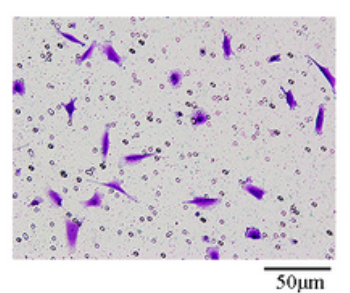

Lv-miR-451-hBMSCs
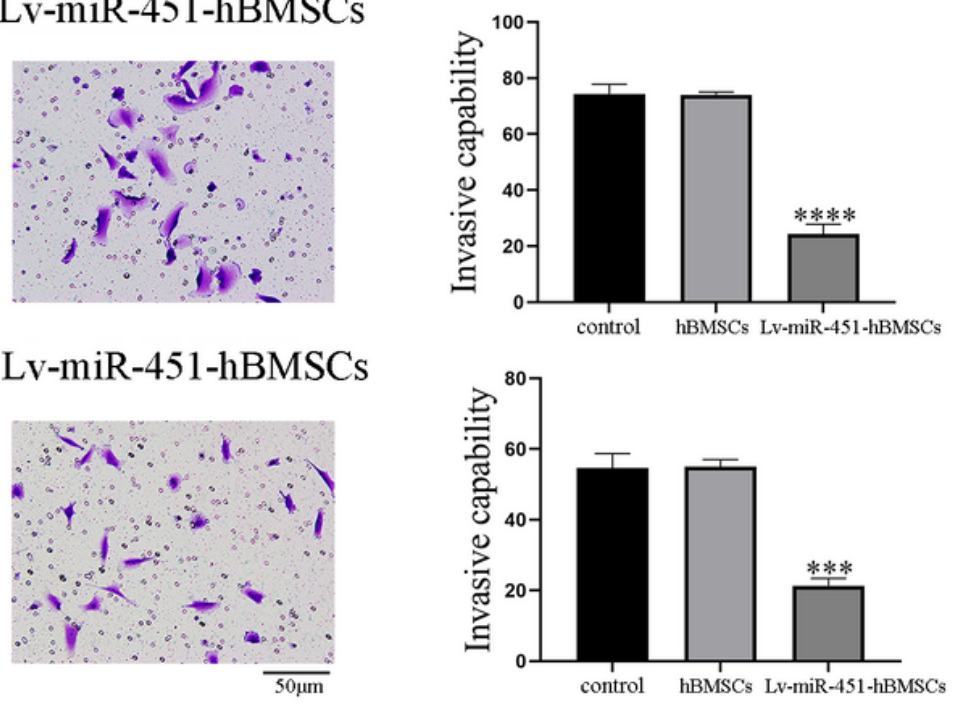

\section{Figure 3}

The culture medium of miR-451-loaded hBMSCs inhibits invasion and migration of glioma cells. (A-C) The invasion ability of glioma cells was evaluated via Transwell assay. (D) The migration ability of glioma cells was evaluated via wound scratch assay. (The results were measurement data, which were expressed as mean \pm standard deviation. The experiment was repeated for three times independently.) ${ }^{\star} \mathrm{P}<0.05,{ }^{*} \mathrm{P}<0.01,{ }^{*} * \mathrm{P}<0.001$ and ${ }^{* \star * \star P} \mathrm{P}<0.0001$. 
A

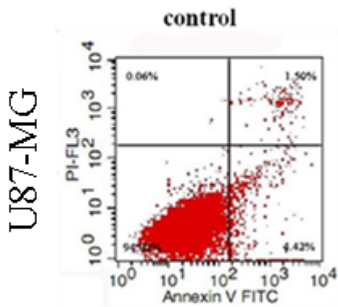

B

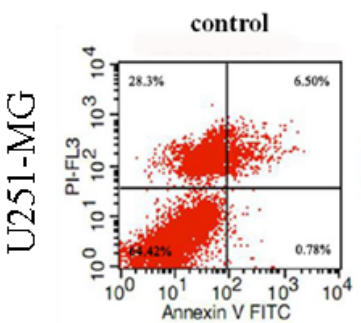

C

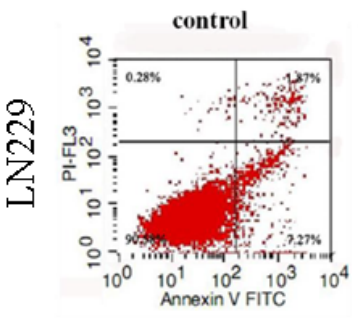

D

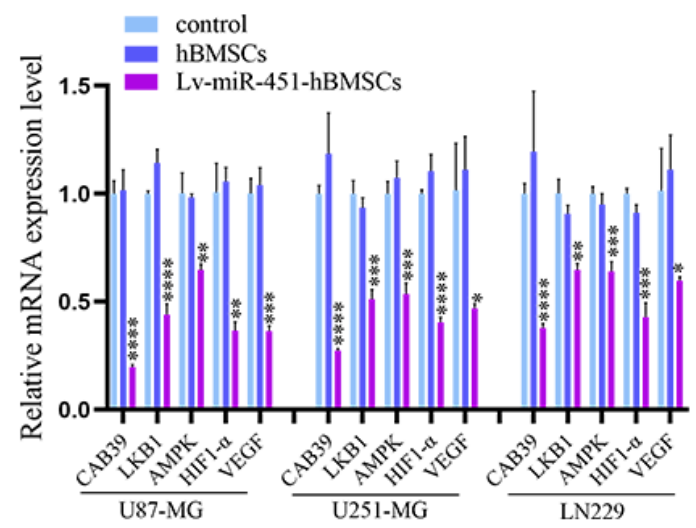

F
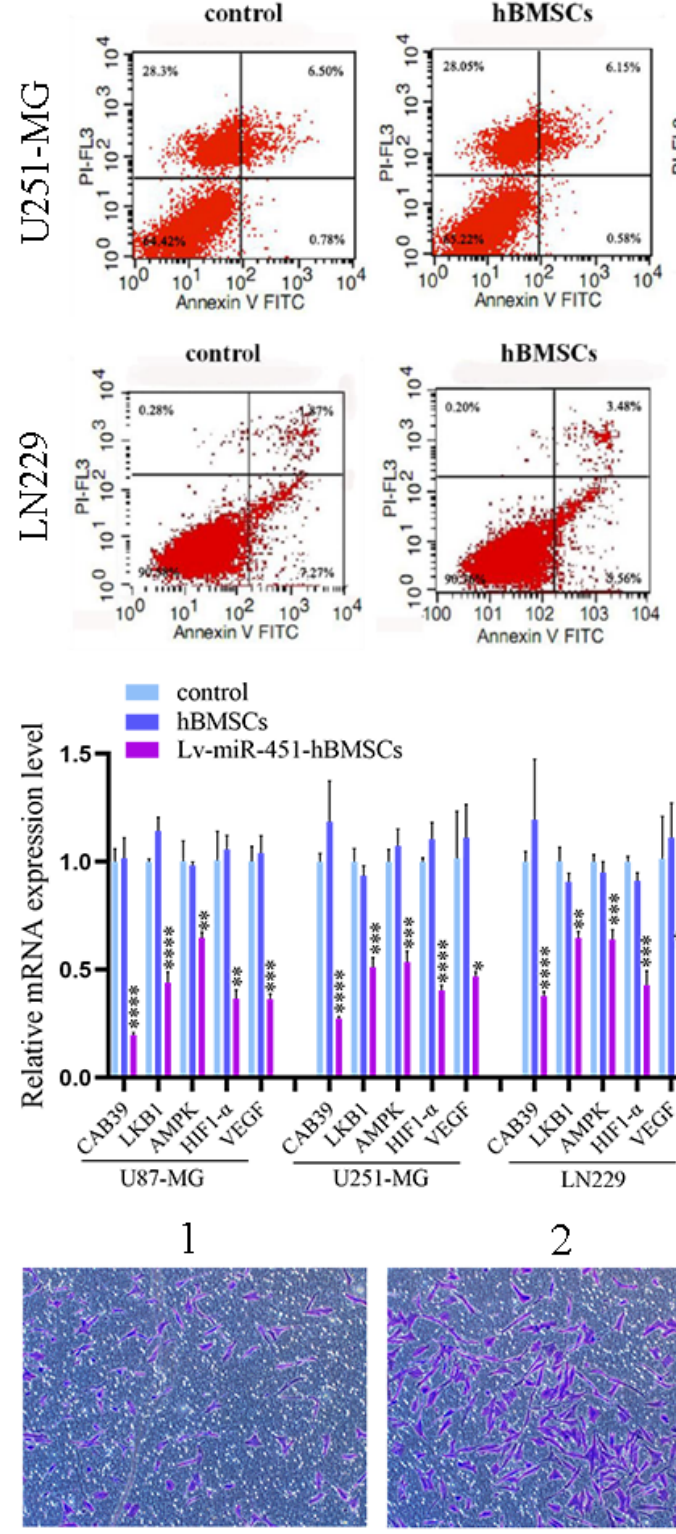

2

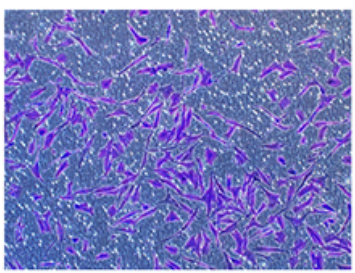

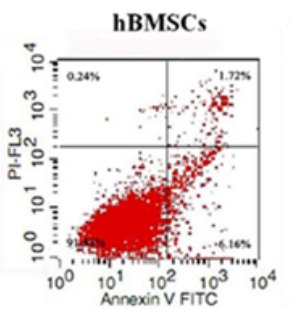

hBMSCs
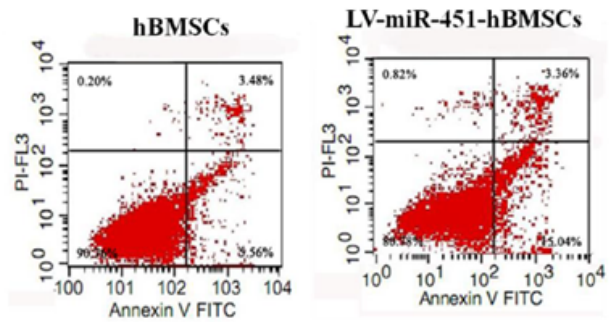

LV-miR-451-hBMSCs

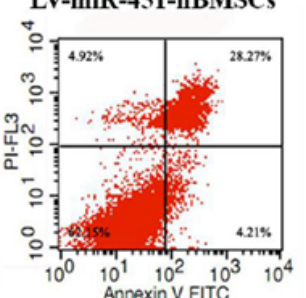

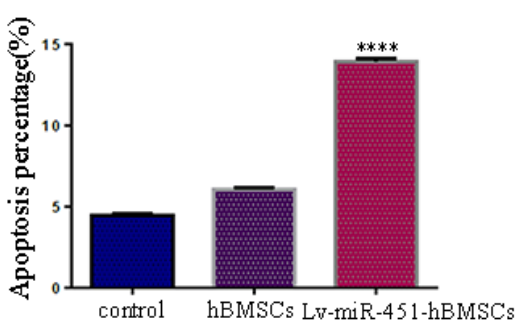
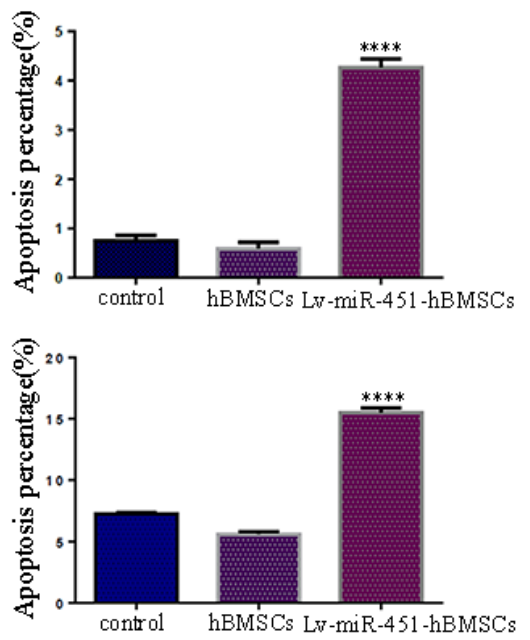
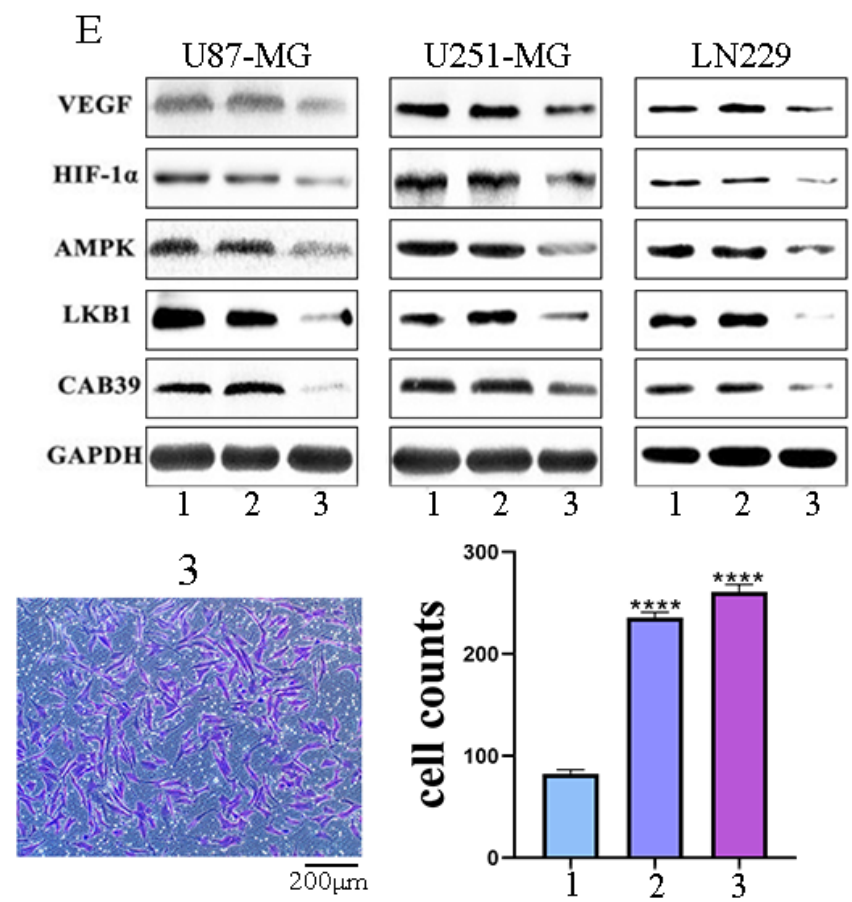

\section{Figure 4}

The culture medium of miR-451-loaded hBMSCs induced apoptosis on glioma cell via LKB1/AMPK/PI3K/AKT pathway. (A-C) Cell apoptosis rate from different treatment groups was analyzed via Annexin V-PI test. (D) RT-PCR analysis of CAB39, LKB1, AMPK, HIF1-a and VEGF mRNA expression levels in U87-MG, U251-MG and LN229 glioma cells in different treatment groups. (E) Protein levels of U87-MG, U251-MG and LN229 cells treated with (1) the normal culture medium, (2) the culture medium of hBMSCs, (3) the culture medium of miR-451-loaded hBMSCs. (F) Transwell assay was used to verify the 
tumor chemotaxis of hBMSCs towards conditioned medium from the glioma cell line, U87-MG (1) hBMSCs were loaded in the upper well, and cell-free medium was loaded in the lower well; (2) hBMSCs were loaded in the upper well, and culture medium of U87-MG cells was loaded in the lower well; (3) miR451-loaded hBMSCs were loaded in the upper well, and culture medium of U87-MG cells was loaded in the lower well. ${ }^{*} P<0.05, * \star P<0.01, * \star \star P<0.001$ and $* \star \star \star P<0.0001$.

A

day $0 \quad$ day $7 \quad$ day 14 day $21 \quad$ day 28 day 35 day 42
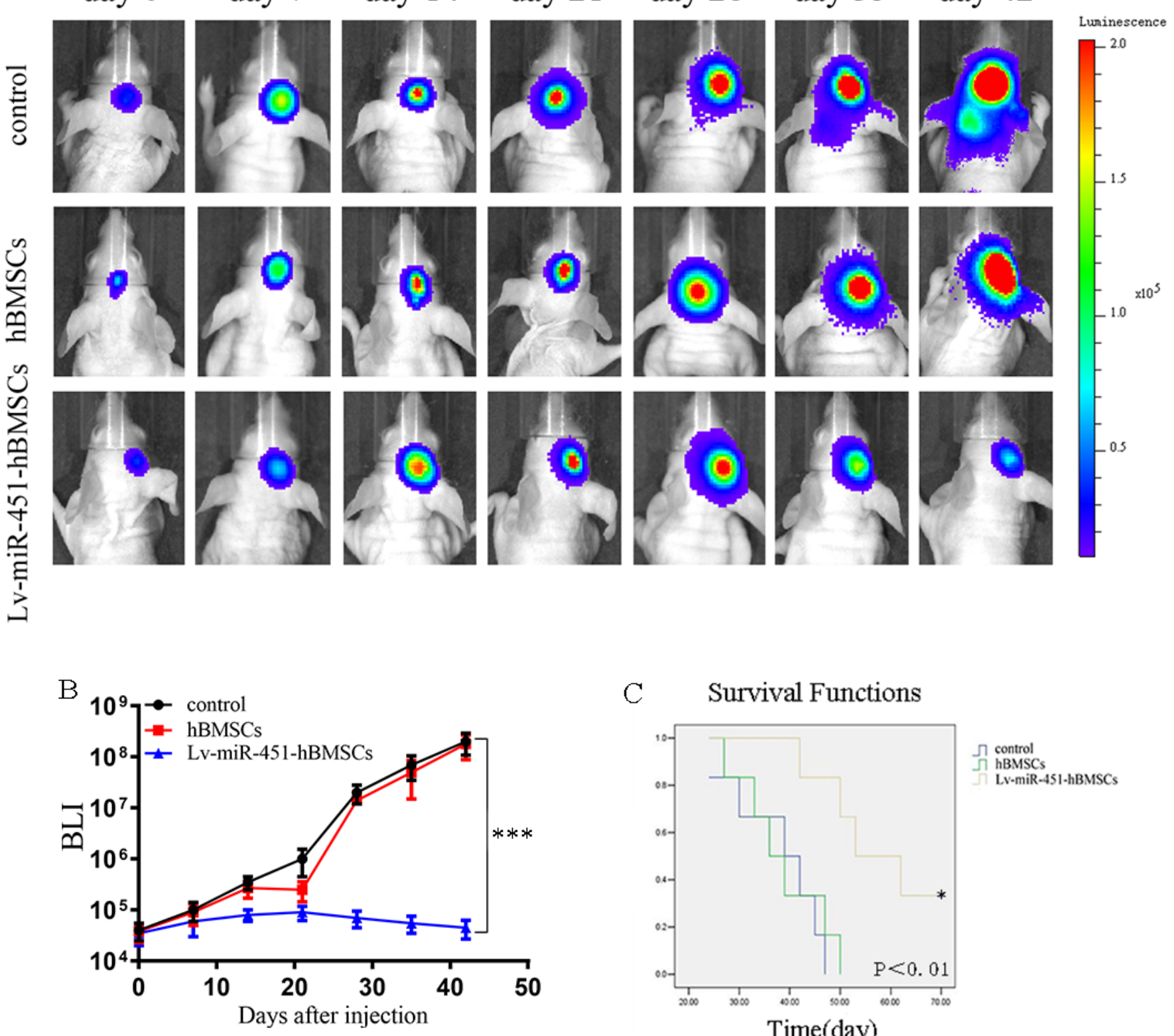

C Survival Functions

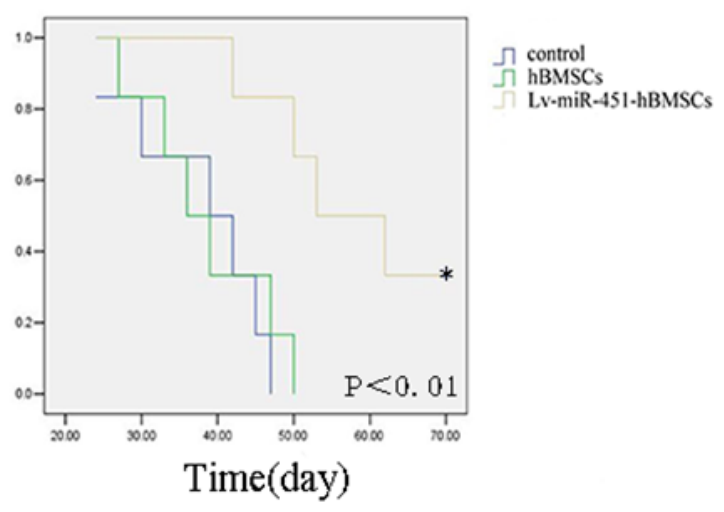

\section{Figure 5}

Efficacy of miR-451-loaded hBMSCs for brain tumor therapy. Bioluminescence images (A) and BLI (B) of the glioma-bearing mice 7, 14, 21, 28, 35, and 42 days after treating with hBMSCs or miR-451-loaded 
hBMSCs. ${ }^{* *} \mathrm{P}<0.001$ (ordinary two-way ANOVA). (C) Survival rate of the glioma-bearing mice after treating with hBMSCs or miR-451-loaded hBMSCs. * $p<0.05$ (Logrank (Mantel-Cox) test).

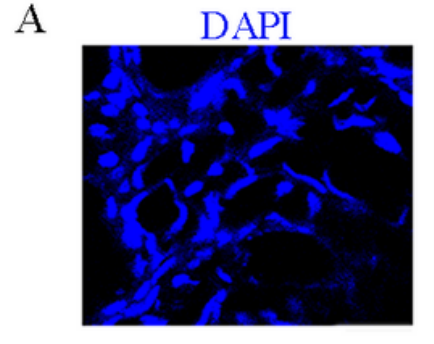

$\mathrm{C}$

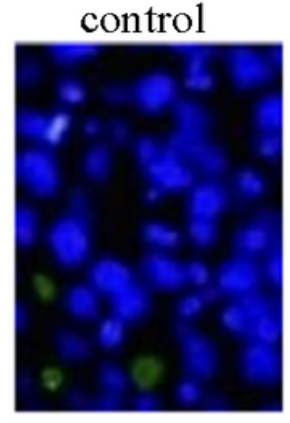

$\mathrm{D}$
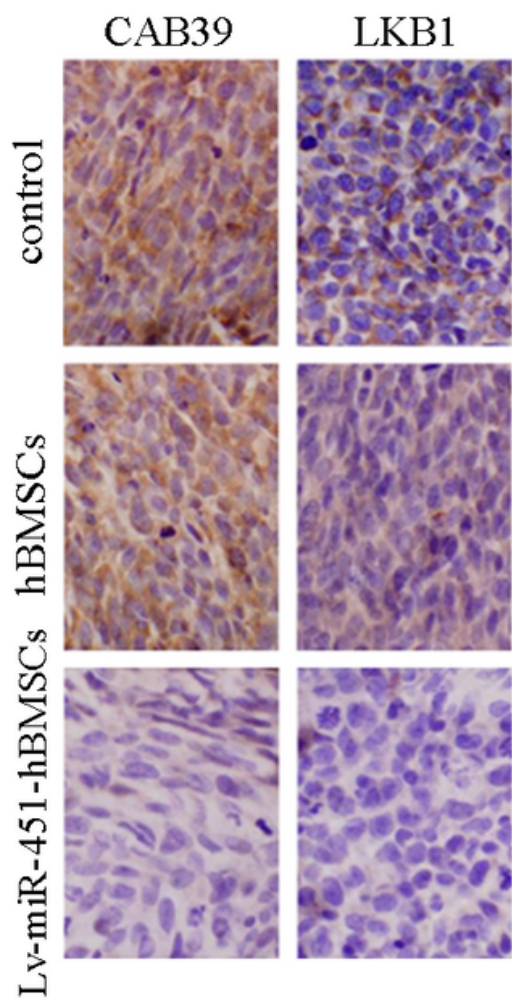

GFP

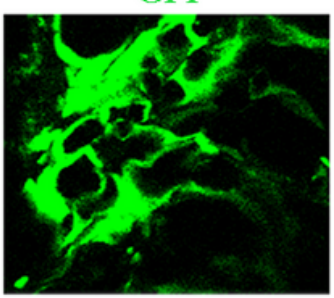

hBMSCs

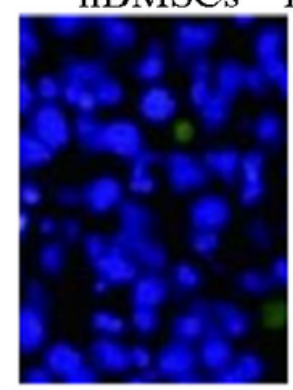

AMPK
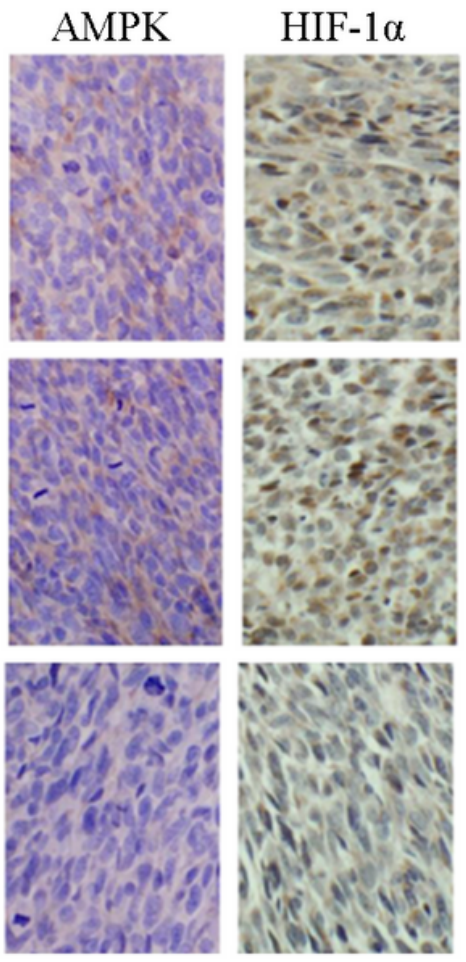

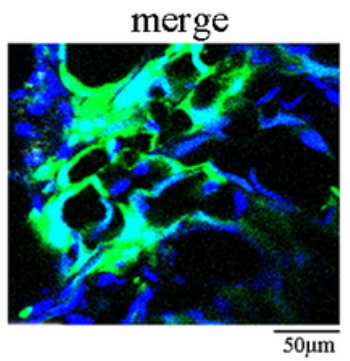

v-miR-451-hBMSCs

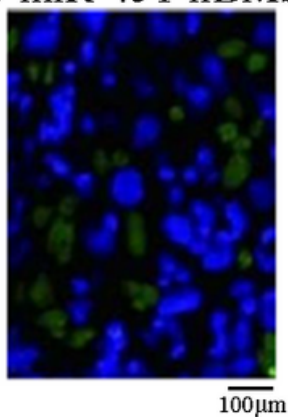

$\mathrm{B}$

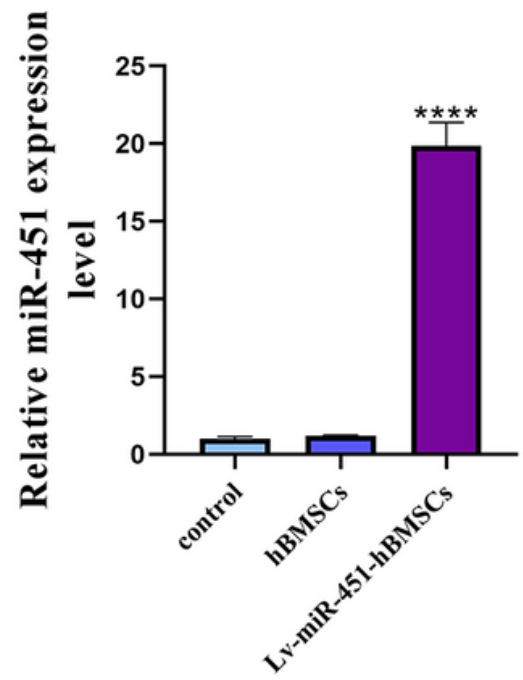

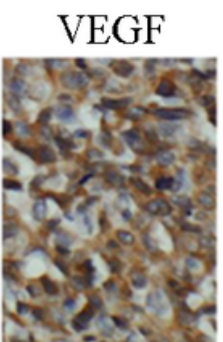

Ki67
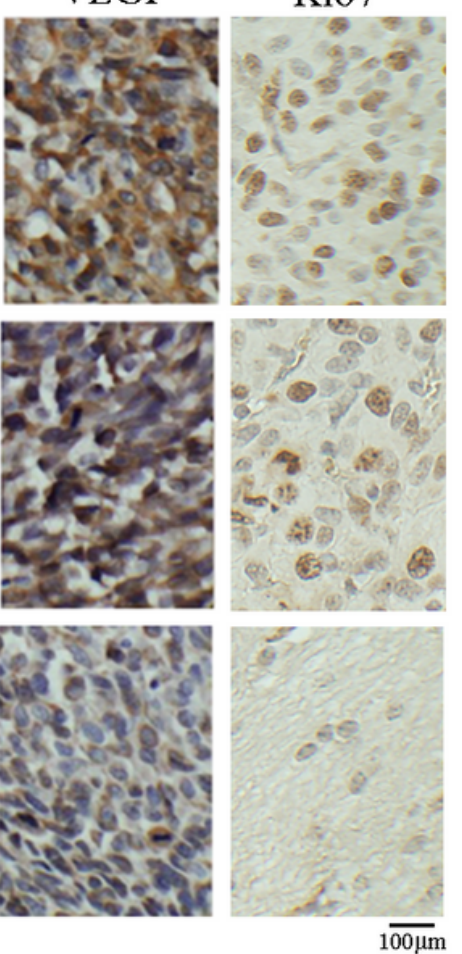

\section{Figure 6}

miR-451-loaded hBMSCs inhibits tumor growth in mice by upregulating the expression of miR-451. (A) Representative images of EGFP-expressing hBMSCs in tumor tissue slices after $24 \mathrm{~h}$ injection. Nuclei were stained with DAPI. (B) The expression of miR-451 in tumor tissues was evaluated with RT-qPCR. ${ }^{\star * \star}$ p $<$ 0.0001 compared with the control group. (C) Representative photographs of TUNEL staining in tumor 
tissue from different treatment groups. (D) Immunohistochemistry detection for CAB39, LKB1, AMPK, HIF1a, VEGF and Ki67 in tumor tissue from different treatment groups.

\section{Supplementary Files}

This is a list of supplementary files associated with this preprint. Click to download.

- SupplementaryFiles.pdf 